\title{
Adverse Event MedDRA Low Level Term Code
}

National Cancer Institute

\section{Source}

National Cancer Institute. Adverse Event MedDRA Low Level Term Code. NCI Thesaurus.

Code C117383.

A coded value specifying the low level term for the adverse event from the Medical

Dictionary for Regulatory Activities (MedDRA). 